Article

\title{
Calculating Point Cloud Object Volume using Co- Opposite-Direction Slicing Method
}

\author{
Bin Li ${ }^{1, *}$, Xiaowei Bi ${ }^{1}$, Cheng Peng ${ }^{1}$, Yong Chen ${ }^{2}$, Xiaofa Zhao ${ }^{3}$ and Chengsheng Yang ${ }^{1, *}$ \\ 1 Dept. of Geomatics, School of Geology Engineering and Geomatics, Chang'an University, Xian \\ 710054, China; binliok@chd.edu.cn (B.L.); 594802727@qq.com (X.B.); 2998593278@qq.com (C.P.); \\ 86294891@qq.com (C.Y.) \\ 2 Hebei Provincial Geological Environment Monitoring Institute, Shijiazhuang 050022, China; \\ 150128282@qq.com (Y.C.) \\ 3 Shenzhen Cadastral Surveying and Mapping Office, Shenzhen 518000, China; 21968322@qq.com \\ (X.Z.) \\ * Correspondence: binliok@chd.edu.cn (B.L.), +86-1862-908-2897; 86294891@qq.com (C.Y.), Tel.: +86- \\ 1348-826-5004 (C.Y.)
}

\begin{abstract}
Although the Slicing Method (SM) is effective for calculating the volume of point cloud objects (PCOs), it is restricted in terms of applicability and practicability because of a certain contingency and directional defects. The Co-Opposite-Direction Slicing Method (CODSM) proposed in this paper is an improved method for calculating PCO volume by increasing parallel (co-opposite-direction) observation and considering the two-way mean as the result. This method takes full advantage of the mutual offsetting of random errors and the compensation of systematic directional errors, which can effectively overcome (or mitigate) the effect of random errors and reduce the effect of systematic errors in SM. In this paper, two typical objects, a cone model and a stone lion base, are the examples for calculating PCO volume using CODSM. The results show that CODSM has all the inherent advantages of SM and effectively weakens the volatility of random errors and the directionality of systematic errors from SM. Therefore, CODSM is a robust configuration upgrade of SM.
\end{abstract}

Keywords: point cloud objects (PCOs); volume calculation (VC); co-opposite-direction slicing method (CODSM); systematic directional errors; random errors

\section{Introduction}

The volume calculation of PCOs (PCVC) is an effective way to obtain the volume of spatial objects [1-4], and is being widely applied in fields such as forestry, geology, engineering, transportation, navigation, and surveying and mapping [5-10]. Although a variety of PCVC methods are available [11,12], only two, namely segmenting [13] and slicing [14], can be considered precise and effective for irregular objects.

The traditional segmenting method consists of six steps [13], whereas the slicing method originating from it is simplified to five steps [15]. The main difference between these two methods is the lack of the projection link in the segmenting method. Without this projection link, the slicing method can avoid the inherent triple magnifying effects and the possible shape distortion effect commonly seen in the segmenting method [15]. However, since the shape of the slice varies with the slicing position, the volume calculation base of the single shape is bound to change accordingly, which distorts the final volume calculation results. Owing to these problems and pitfalls, the adaptability, reliability and robustness of the slicing method is affected and questioned in practical application which, to a certain extent, restricts its popularization and use. To effectively deal with the volume calculation distortion incurred by random errors and directionality of the slicing method, improvements are necessary in terms 
of ensuring that its theoretical model and algorithm better satisfy the requirements of practical application and engineering of PCVC.

Based on these facts and existing SM, this paper makes use of the mutual compensation of random errors and the directional consistency of systematic errors [16] and proposes the CoOpposite-Direction Slicing Method (CODSM), which increases parallel observations and uses the two-way mean as the result, for PCVC. As an improved method, CODSM tries to foster the strengths of the original method-maintaining the advantage of high efficiency, while circumventing the weaknesses - and eliminating shortcomings such as weak resistance against random errors and insufficient consideration of systematic directional errors. To verify the effectiveness of this algorithm, the focus of this paper was a PCVC experiment with CODSM by considering two working conditions for each of the two typical objects - a cone model and a stone lion base-to analyze and compare the results of CODSM with traditional SM. The results indicate that the CODSM proposed in this paper can effectively alleviate or even overcome the double adverse effects of error contingency and directionality. CODSM is therefore not only an upgrade for SM, but also a full-fledged practical and effective method for PCVC.

\section{Methods}

CODSM is derived from SM. The fundamental principles of CODSM in the forward (topdown) and reverse (bottom-up) directions for a laser scanned PCO which is sliced horizontally are summarized in the process shown in Figure 1. As shown in the figure, the PCO data $D$ can be obtained by object scanning; between the longitudinal minimum value 0 and maximum value $H$ of the PCO, a set of $(n+1)$ slices $S_{i}$ (as shown in equation (2)) with equal spacing $(h$, as shown in equation (1)) in the horizontally forward or reverse directions can be obtained by forward or reverse slicing. The outline boundary is determined by the outline boundary polygon $P_{i}(i=0,1, \ldots, m)$. Calculating the area of polygon $A_{i}(i=0,1, \ldots, n)$, as shown in equation (3), gives the area of the slices. The forward or reverse PCO volume $V_{j}(j=1,2)$ can then be obtained by accumulating the bottom area $A_{i}$ and then multiplying by $h$, as shown in equation (4), where $V_{1}$ and $V_{2}$ are the forward and reverse slicing values of PCO volume, respectively, while the mean value $V$, as shown in equation (5), is the PCO volume calculated by CODSM.

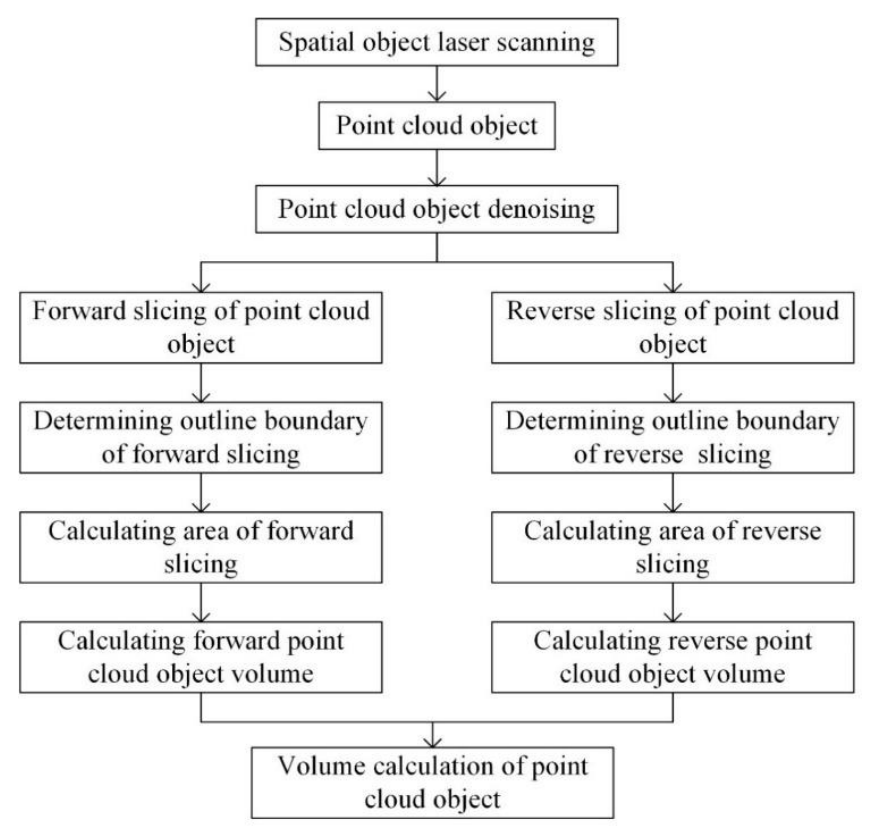


Figure 1. Flow chart of point cloud object volume calculation with opposite-direction slicing method.

$$
\begin{gathered}
h=\frac{H}{n} \\
S_{i}=\left\{\mathrm{D}(\mathrm{x}, \mathrm{y}, \mathrm{z}) \mid \mathrm{x} 、 \mathrm{y} \in \mathrm{R}+, \mathrm{z}=\frac{i}{n} * H_{,} i=0, \ldots, n\right\} \\
A_{i}=\frac{1}{2} \sum_{j=0}^{m-1}\left|\begin{array}{cc}
x_{j} & y_{j} \\
x_{j+1} & y_{j+1}
\end{array}\right| \\
V_{j}=\sum_{i=0}^{n-1} A_{i} * h \\
V=\frac{1}{2}\left(V_{1}+V_{2}\right)
\end{gathered}
$$

In the above equations, $H$ is the height of PCO; $n+1$ is the total number of forward or reverse slices; $m$ is the number of polygonal vertices of the slice outline boundary; $x, y, z$ are the polygonal vertex coordinates of the slice outline boundary; $V_{1}$ represents the forward PCO volume; and $V_{2}$ represents the reverse PCO volume. The traditional slicing [14] is a one-way $\mathrm{SM}$. For basic slices, the shape is related to the slicing position (value $z$ ), and because each slice is surrounded by adjacent slices, the single volume varies with the slice and the slicing position. In other words, different slicing directions and spacings may produce different calculation results of $V_{1}$ and $V_{2}$ with SM. The CODSM, by taking the mean value of $V_{1}$ and $V_{2}$ as the optimal estimation, can objectively eliminate random errors and may also reduce directional systematic errors, thus improving the reliability and precision of PCVC results. In other words, the CODSM based on the principle of "mean eliminating error" $[16,17]$ has the advantage of complementary gains and losses, and thus can be considered a simple and effective PCVC method.

\section{Experimental Model Design}

To verify the effectiveness of CODSM, the experiment was designed and conducted using the highest possible PCO scanning resolution $(1 \mathrm{~mm})$ and the most complete slicing spacing ( $2^{n} \mathrm{~mm}, n=0,1,2,3, \ldots$ ). This experiment is based on the PCO data (as shown in Figures 2c and 3c) of two objects, i.e., a regular cone model (shown in Figure $2 b$ ) and an irregular stone lion base (shown in Figure 3a). These objects were chosen to meet the requirements for "verifying the correctness and applicability" and representativeness. The cone is a regular object whose geometric dimensions can be measured and used to calculate the volume accurately [14] (as shown in Figure 2a), and therefore can be used to verify the correctness of the algorithm whereas the stone lion base is an irregular object with moderate complexity whose volume cannot be accurately measured or calculated (as shown in Figure 3c), and therefore can verify the applicability of the algorithm as a general example [15]. Figure 2a shows the precise measurement results of the geometric dimensions of the cone model [14] that can be used to calculate the approximate truth-value of the cone volume [15]. The radius of the base circle of the cone is $78.0 \mathrm{~mm}$ and the cone height is $188.4 \mathrm{~mm}$. 


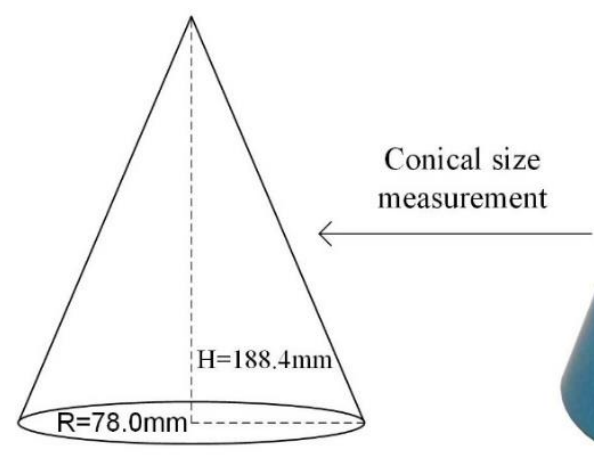

(a)

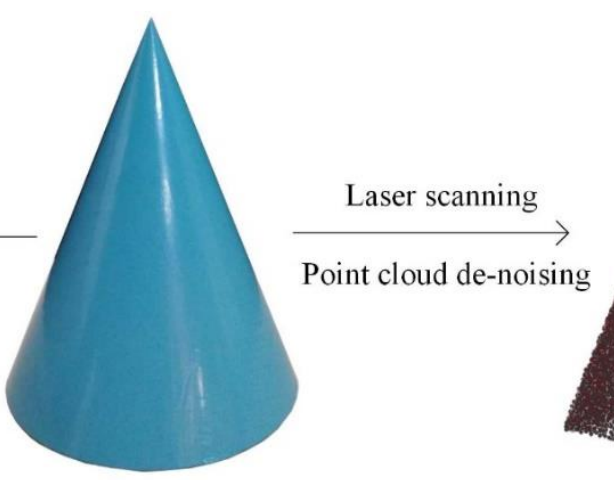

(b)

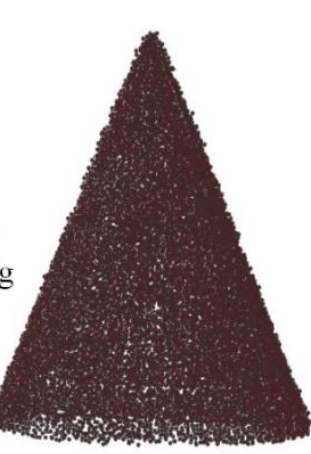

(c)

Figure 2. Cone model, its size and point cloud data. (a) Measuring the radius and height of the cone model; (b) The cone model; (c) Point cloud data of the cone model.

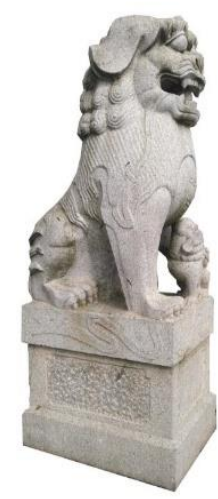

(a)

\section{Laser scanning \\ Point cloud de-noising}

(a)

Figure 3. Physical map and point cloud data of the stone lion base. (a) The stone lion; $(b)$ Point cloud data of the stone lion; (c) Point cloud data of the stone lion base.

\subsection{Slicing Spacing Design}

For the purpose of this experiment, it has been stipulated that the slicing spacing (hereinafter referred to as spacing) is $h$, the forward direction is from top to bottom, and the reverse direction is from bottom to top.

The value of $h$ is set to satisfy the requirements of equation (6). When forward or reverse slicing is performed between the longitudinal minimum value 0 and the maximum value $H$ of the PCO, $n+1$ slices $S_{i}(i=0,1,2,3, \ldots, n)$ can be obtained. Since $H$ is generally not divisible by $h$, there are $n$ equally spaced slices with the spacing of $h$ and 1 slice with a height less than $h$ (set to $h_{b}$ ). $h_{b}<h$ and the segment corresponding to $h_{b}$ is identified as the remnant of PCO (hereinafter referred to as PCO remnant).

$$
h=2^{k} \leq H
$$

Here $k \in(0,1,2,3,4), h \leq H, h_{b}<h$.

When choosing the value of the slicing spacing $h$ on the experiment scale, both the precision of the estimation and representativity of the span were considered.

\subsection{Volume Calculation Experiment}


During the one-way forward or reverse PCO volume experiment of SM, the volume $V$ is composed of two parts - the main volume $V_{a}$ and the remnant volume $V_{b}$, which can be calculated as shown in equations (7)-(9). $V_{a}$ corresponds to the main part of PCO with a height of $h *(n-1)$, which can be divided by spacing $h$, while $V_{b}$ is the PCO remnant with a height of $h_{b}$.

$$
\begin{gathered}
V_{a}=\sum_{i=1}^{n-1} A_{i} * h \\
V_{b}=A_{n} * h_{b} \\
h_{b}=(H-h *(n-1)) \\
V=V_{a}+V_{b}
\end{gathered}
$$

Equations (7)-(10) are suitable for calculating the volumes of both forward and reverse PCOs, and their mean value is the final PCO volume as calculated by CODSM.

\section{Results}

Taking the regular cone (shown in Figure 4) and the irregular stone lion base (shown in Figure 5) as examples, the experiment was conducted along their positive and oblique axes, respectively, followed by denoising [18-20] and calculation [14] of the PCO data for the laser scanned objects to verify the compensation, applicability and correctness of CODSM by both representative and general experiments.

\subsection{Biaxial Experiment on the Cone}

As there is forward and reverse axisymmetry along the horizontal axis of the cone (i.e., the horizontal cone has upper and lower symmetry with the horizontal axis as the symmetry axis, as shown in Figures 4c and 4d), the selected and listed experiment calculations and analyzed data are the experimental results of the representative positive axis and general oblique axis of the cone (as shown in Tables 1 and 2 and Figures 6 and 7). Based on these results, the analysis and comparison of the characteristics of one-way and co-opposite-direction algorithms can be performed. 

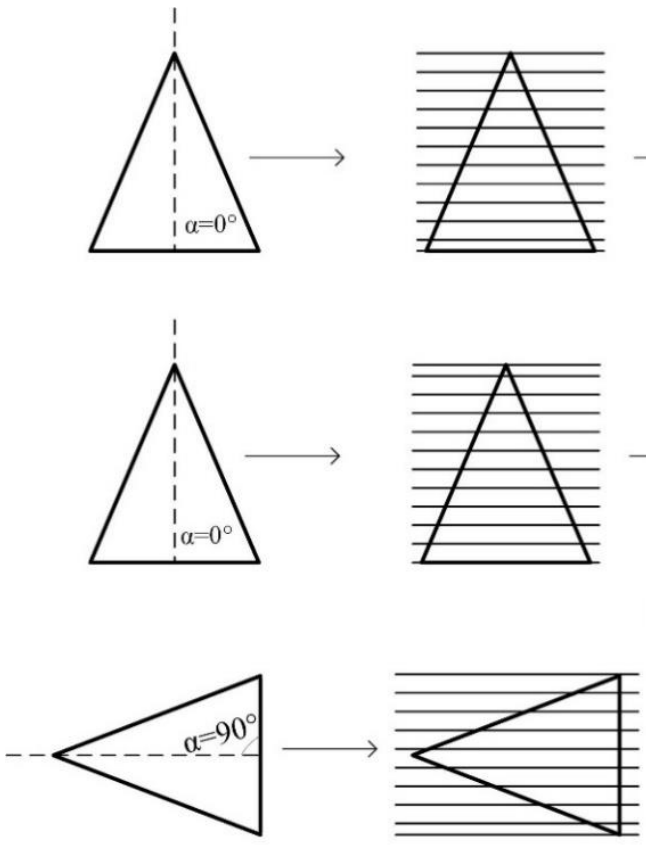

()

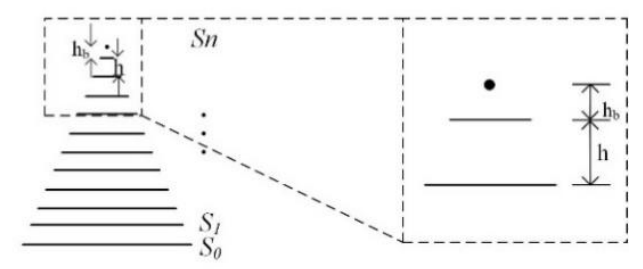

(b)

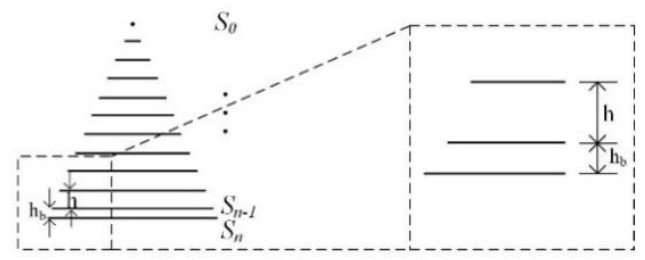

)

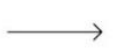

b)

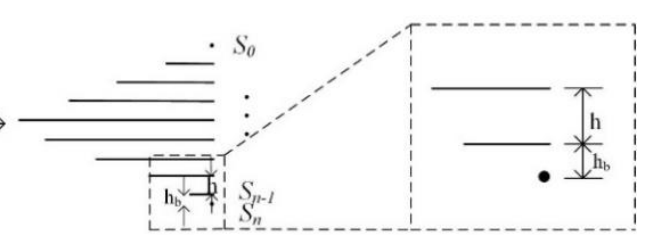

(c)
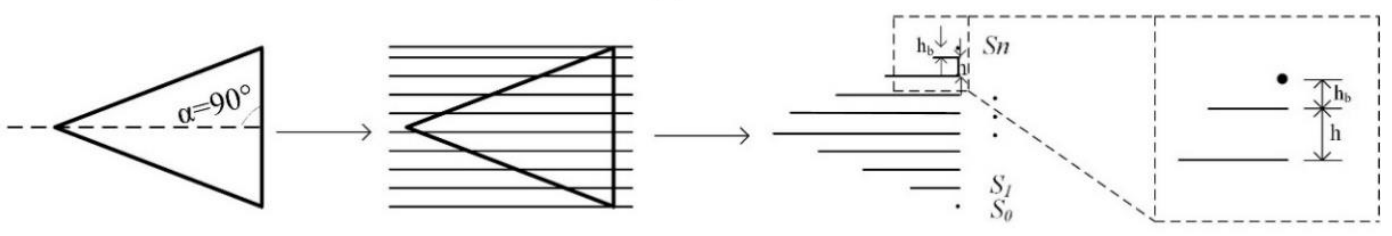

(d)
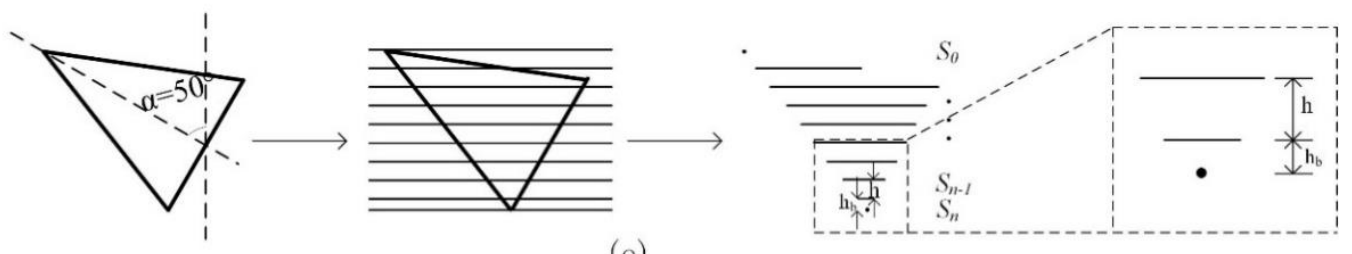

(e)
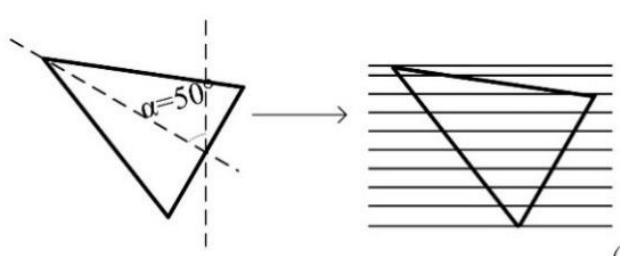

(f)

Figure 4. Schematic of the triaxial positive and negative cutting form of conical point cloud. (a) Sectional drawing of a normal positive axis; (b) Sectional drawing of a normal negative axis; (c) Sectional drawing of a transverse positive axis; (d) Sectional drawing of a transverse negative axis; (e) Sectional drawing of an oblique positive axis; (f) Sectional drawing of an oblique negative axis. 

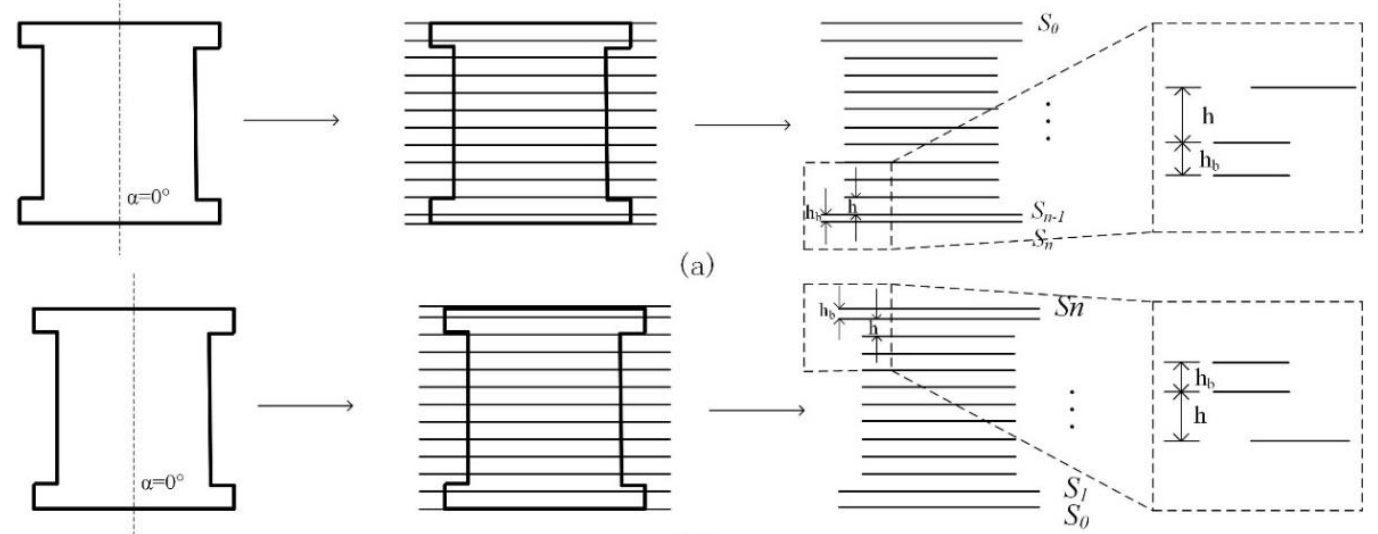

(b)
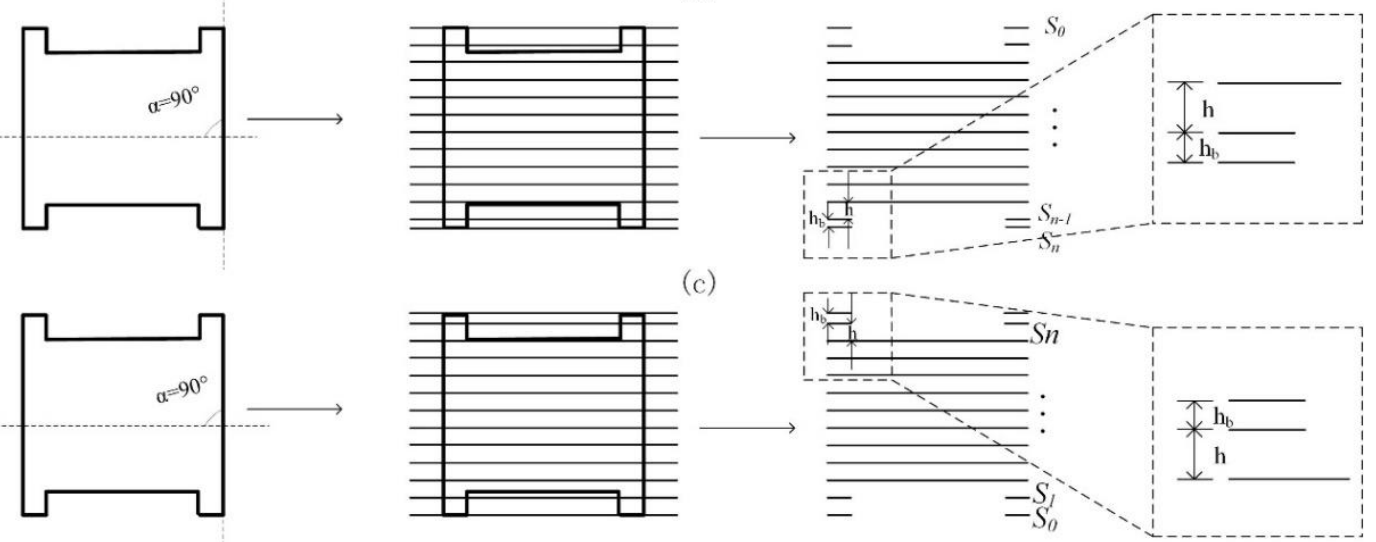

(d)
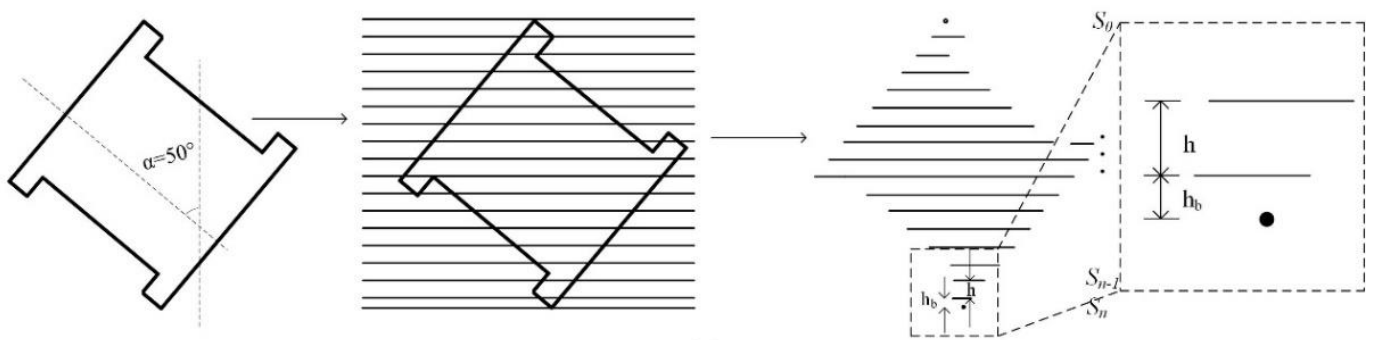

(e)
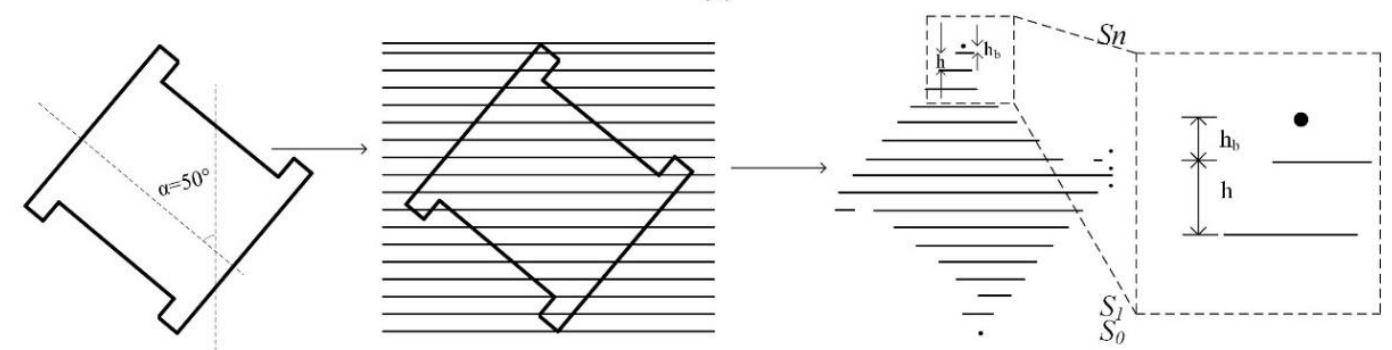

(f)

Figure 5. Schematic of test cutting form of oblique axis of stone lion base point cloud. (a) Sectional drawing of a normal positive axis; (b) Sectional drawing of a normal negative axis; (c) Sectional drawing of a transverse positive axis; (d) Sectional drawing of a transverse negative axis; (e) Sectional drawing of an oblique positive axis; (f) Sectional drawing of an oblique negative axis.

\subsubsection{Positive Axis Experiment of a Cone}


According to the positive axis SM shown in Figures $4 \mathrm{a}$ and $4 \mathrm{~b}$, spacings of $1 \mathrm{~mm}, 2 \mathrm{~mm}, 4$ $\mathrm{mm}, 8 \mathrm{~mm}$, and $16 \mathrm{~mm}$ have been adopted to calculate the one-way and co-opposite-direction forward and reverse PCO volumes along the positive axis of cone (as shown in Table 1). As the true volume of cone can be calculated [14] $\left(473,142,270 \mathrm{~mm}^{3}\right)$, the one-way and co-oppositedirection volumes and their relative errors can be calculated under different conditions; the results and trend are shown in Table 1 and Figure 6, respectively.

Table 1. Comprehensive experimental results for normal axis of cone model.

\begin{tabular}{|c|c|c|c|c|c|}
\hline Method & $\begin{array}{l}\text { Spacing } \\
(\mathrm{mm})\end{array}$ & $\begin{array}{l}\text { Volume } \\
\left(\mathrm{mm}^{3}\right)\end{array}$ & $\begin{array}{l}\text { Absolute error } \\
\qquad\left(\mathrm{mm}^{3}\right)\end{array}$ & $\begin{array}{l}\text { Relative } \\
\text { error(\%) }\end{array}$ & $\begin{array}{l}\text { Number } \\
\text { of Slices }\end{array}$ \\
\hline Positive & 1 & 473138434 & 0 & 0 & 841 \\
\hline \multirow[t]{4}{*}{ Slicing } & 2 & 473140926 & 2492 & 0.0005267 & 421 \\
\hline & 4 & 473262638 & 124204 & 0.02625109 & 211 \\
\hline & 8 & 473779636 & 641202 & 0.13552101 & 106 \\
\hline & 16 & 473973105 & 834671 & 0.17641158 & 53 \\
\hline Negative & 1 & 473138559 & 125.5 & 2.6525E-05 & 841 \\
\hline \multirow[t]{4}{*}{ Slicing } & 2 & 473135488 & -2946 & -0.0006227 & 421 \\
\hline & 4 & 473101818 & -36616 & -0.007739 & 211 \\
\hline & 8 & 473156822 & 18388 & 0.00388639 & 106 \\
\hline & 16 & 471381018 & -1757416 & -0.371438 & 53 \\
\hline Opposite & 1 & 473138496 & 62.75 & 1.3263E-05 & 1682 \\
\hline \multirow[t]{4}{*}{ Slicing } & 2 & 473138207 & -227 & $-4.798 E-05$ & 842 \\
\hline & 4 & 473182228 & 43794 & 0.00925606 & 422 \\
\hline & 8 & 473468229 & 329795 & 0.0697037 & 212 \\
\hline & 16 & 472677061 & -461372.5 & -0.0975132 & 106 \\
\hline
\end{tabular}

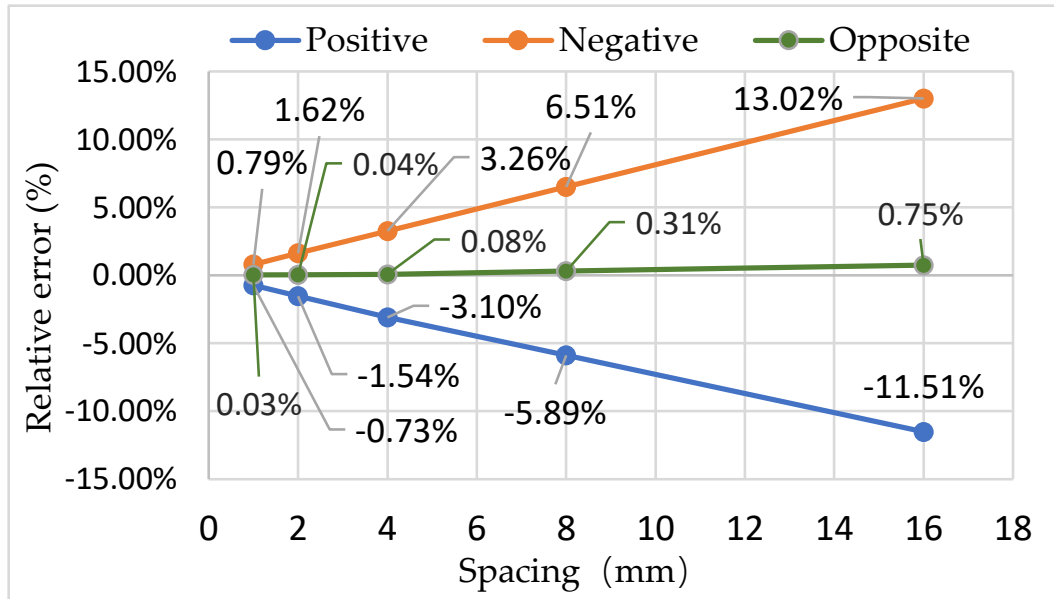

Figure 6. Relative error diagram of volume calculation for normal axis of cone model.

As can be seen from Table 1 and Figure 6, the absolute values of volume errors along the positive axis of the cone in forward and reverse SM are both proportional to the slicing spacing, and the errors are almost the same but with opposite symbols. Thus, it can be inferred that 
larger spacings result in larger the errors. However, the mean value of CODSM offsets the errors caused by the differences in slicing direction.

\subsubsection{Oblique Axis Experiment of a Cone}

To discuss a more general scenario, for example one based on the oblique axis SM shown in Figures $4 \mathrm{e}$ and 4f, spacings of $1 \mathrm{~mm}, 2 \mathrm{~mm}, 4 \mathrm{~mm}, 8 \mathrm{~mm}$, and $16 \mathrm{~mm}$ have been adopted to calculate the one-way and co-opposite-direction forward and reverse PCO volumes along the oblique axis of the cone (as shown in Table 2). For the same cone with a known volume, the volumes and their relative errors are shown in Table 2 and the trend is shown in Figure 7.

Table 2. Comprehensive experimental results for oblique axis of cone model.

\begin{tabular}{cccccc}
\hline Method & $\begin{array}{c}\text { Spacing } \\
(\mathrm{mm})\end{array}$ & $\begin{array}{c}\text { Volume } \\
\left(\mathrm{mm}^{3}\right)\end{array}$ & $\begin{array}{c}\text { Absolute error } \\
\left(\mathrm{mm}^{3}\right)\end{array}$ & $\begin{array}{c}\text { Relative } \\
\text { error }(\%)\end{array}$ & $\begin{array}{c}\text { Number } \\
\text { of Slices }\end{array}$ \\
\hline Positive & 1 & 464882299 & -8256134.5 & -1.7449723 & 1018 \\
Slicing & 2 & 464904633 & -8233800.5 & -1.7402519 & 509 \\
& 4 & 465026217 & -8112216.5 & -1.7145545 & 255 \\
& 8 & 464939421 & -8199012.5 & -1.7328993 & 128 \\
\hline Negative & 1 & 464882626 & -8255808 & -1.7449033 & 1018 \\
Slicing & 2 & 464905286 & -8233147.5 & -1.7401139 & 509 \\
& 4 & 464781286 & -8357147.5 & -1.7663218 & 255 \\
& 8 & 464471468 & -8666965.5 & -1.8318033 & 128 \\
\hline Opposite & 1 & 464093457 & -9044976.5 & -1.9116977 & 64 \\
\hline Slicing & 2 & 464904960 & -8233474 & -1.7401829 & 1018 \\
& 4 & 464903752 & -8234682 & -1.7404382 & 510 \\
& 16 & 464705445 & -8432989 & -1.7823513 & 256 \\
\hline & 463598236 & -9540197.5 & -2.0163649 & 64 \\
\hline & 16 & & -9292587 & -1.9640313 & 128 \\
\hline
\end{tabular}

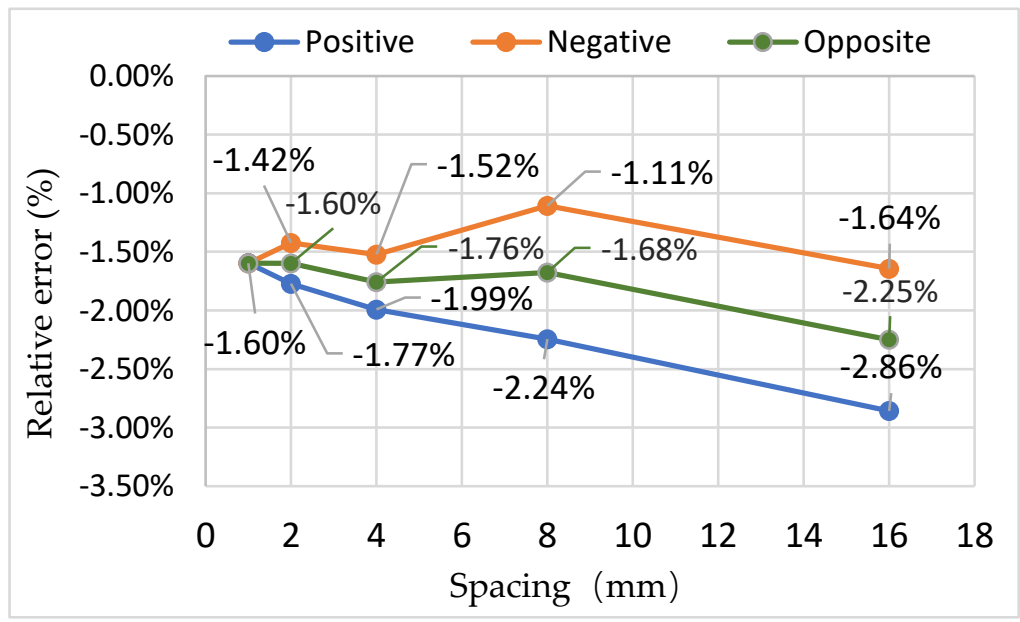

Figure 7. Relative error diagram of volume calculation for oblique axis of cone model. 
As can be seen from Table 2 and Figure 7, although the volume calculation errors along the oblique axis of the cone by forward or reverse SM are roughly proportional to the slicing spacing, the volatility (or contingency) and directional errors have been observed to increase with spacing. However, the mean value of CODSM overcomes (or partially offsets) these contingency and directional errors. This is because the dominant direction of the one-way SM is unknown in advance and the mean error of CODSM ensures that it is smaller than the error of the forward slicing method.

\subsection{Biaxial Experiment of a Stone Lion Base}

As the forward and reverse shapes of a stone lion base are axisymmetric (i.e., there is upper and lower symmetry with the horizontal axis as the symmetry axis, as shown in Figures 5a-5d) along both the positive and horizontal axes, it is sufficient to select a single axis along which to conduct the experiment. The selected and listed experiment calculations, and analyzed data are still the experiment results of representative positive axis and general oblique axis of the stone lion base (as shown in Tables 3 and 4, and Figures 8 and 9). Therefore, the characteristics of one-way and co-opposite-direction algorithms can be analyzed and compared based on these results.

\subsubsection{Positive Axis Experiment of a Stone Lion Base}

According to the positive axis SM shown in Figures $5 \mathrm{a}$ and 5b, spacings of $1 \mathrm{~mm}, 2 \mathrm{~mm}, 4$ $\mathrm{mm}, 8 \mathrm{~mm}$, and $16 \mathrm{~mm}$ have been adopted to calculate the one-way and co-opposite-direction forward and reverse PCO volumes and their relative errors along the positive axis of the stone lion base (as shown in Table 3), and the trend is shown in Figure 8.

Table 3. Comprehensive experimental results for normal axis of stone lion base.

\begin{tabular}{cccccc}
\hline Method & $\begin{array}{c}\text { Spacing } \\
(\mathrm{mm})\end{array}$ & $\begin{array}{c}\text { Volume } \\
\left(\mathrm{mm}^{3}\right)\end{array}$ & $\begin{array}{c}\text { Absolute error } \\
\left(\mathrm{mm}^{3}\right)\end{array}$ & $\begin{array}{c}\text { Relative } \\
\text { error(\%) }\end{array}$ & $\begin{array}{c}\text { Number } \\
\text { of Slices }\end{array}$ \\
\hline Positive & 1 & 1191564 & -8761 & -0.7298857 & 187 \\
Slicing & 4 & 1181869.5 & -18455.5 & -1.5375419 & 94 \\
& 8 & 1163173.5 & -37151.5 & -3.0951201 & 47 \\
& 16 & 1129681.5 & -70643.5 & -5.8853644 & 24 \\
Negative & 1 & 1209853.00 & 9528.00 & 0.79378502 & 187 \\
Slicing & 4 & 1219795.00 & 19470.00 & 1.62206069 & 94 \\
& 8 & 1239483.00 & 39158.00 & 3.26228313 & 47 \\
& 16 & 1356565.00 & 156240.00 & 13.0164747 & 12 \\
\hline \multirow{3}{*}{ Opposite } & 1 & 1200708.5 & 383.5 & 0.03194968 & 374 \\
Slicing & 4 & 1200832.25 & 507.25 & 0.04225939 & 188 \\
& 8 & 1201328.25 & 1003.25 & 0.08358153 & 94 \\
& 16 & 1204061.25 & 3736.25 & 0.31126986 & 48 \\
\hline & & & & -11.510758 & 12 \\
\hline
\end{tabular}




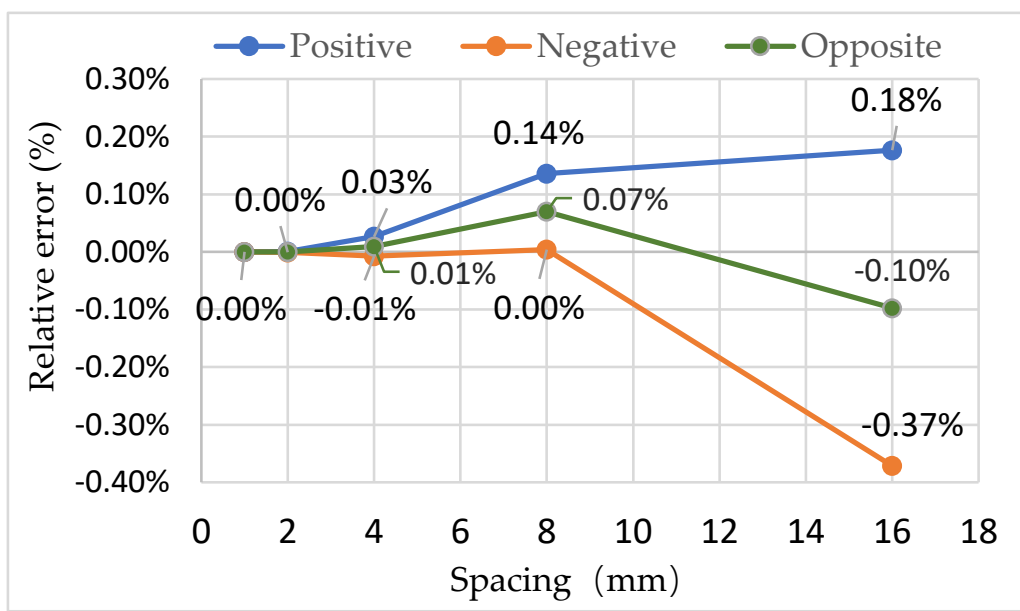

Figure 8. Relative error diagram of volume calculation for normal axis of stone lion base.

As can be seen from Table 3 and Figure 8, although the volume calculation errors of the positive axis of the stone lion base by forward or reverse SM are roughly proportional to the spacing, volatility and directionality have been observed to increase with the spacing. Hence, despite the fact that the forward and reverse compensatory effect of the CODSM with the characteristic of mean value can be shown in each spacing, it is obvious that the smaller the spacing, the better the effect.

\subsubsection{Oblique Axis Experiment of a Stone Lion Base}

Spacings of $1 \mathrm{~mm}, 2 \mathrm{~mm}, 4 \mathrm{~mm}, 8 \mathrm{~mm}$, and $16 \mathrm{~mm}$ have been adopted for calculation of the one-way and co-opposite-direction forward and reverse PCO volumes and their relative errors along the oblique axis of a stone lion base (as shown in Table 4) based on the oblique axis SM shown in Figures 5a and 5b; the trend is shown in Figure 9.

Table 4. Comprehensive experimental results for oblique axis of stone lion base.

\begin{tabular}{cccccc}
\hline Method & $\begin{array}{c}\text { Spacing } \\
(\mathrm{mm})\end{array}$ & $\begin{array}{c}\text { Volume } \\
\left(\mathrm{mm}^{3}\right)\end{array}$ & $\begin{array}{c}\text { Absolute error } \\
\left(\mathrm{mm}^{3}\right)\end{array}$ & $\begin{array}{c}\text { Relative } \\
\text { error(\%) }\end{array}$ & $\begin{array}{c}\text { Number } \\
\text { of Slices }\end{array}$ \\
\hline \multirow{4}{*}{ Positive } & 1 & 1181165.50 & -19159.50 & -1.5961927 & 185 \\
Slicing & 4 & 1179055.00 & -21270.00 & -1.7720201 & 93 \\
& 8 & 1176420.00 & -23905.00 & -1.991544 & 47 \\
& 16 & 1173388.00 & -26937.00 & -2.2441422 & 24 \\
& 1 & 1181150.50 & -19174.50 & -1.5974424 & 185 \\
Negative & 2 & 1183238.50 & -17086.50 & -1.4234895 & 93 \\
Slicing & 4 & 1182037.50 & -18287.50 & -1.5235457 & 47 \\
& 8 & 1187041.50 & -13283.50 & -1.1066586 & 24 \\
& 16 & 1180601.00 & -19724.00 & -1.6432216 & 12 \\
\hline \multirow{3}{*}{ Opposite } & 1 & 1181158.00 & -19167.00 & -1.5968175 & 370 \\
Slicing & 2 & 1181146.75 & -19178.25 & -1.5977548 & 186 \\
& 4 & 1179228.75 & -21096.25 & -1.7575448 & 94
\end{tabular}




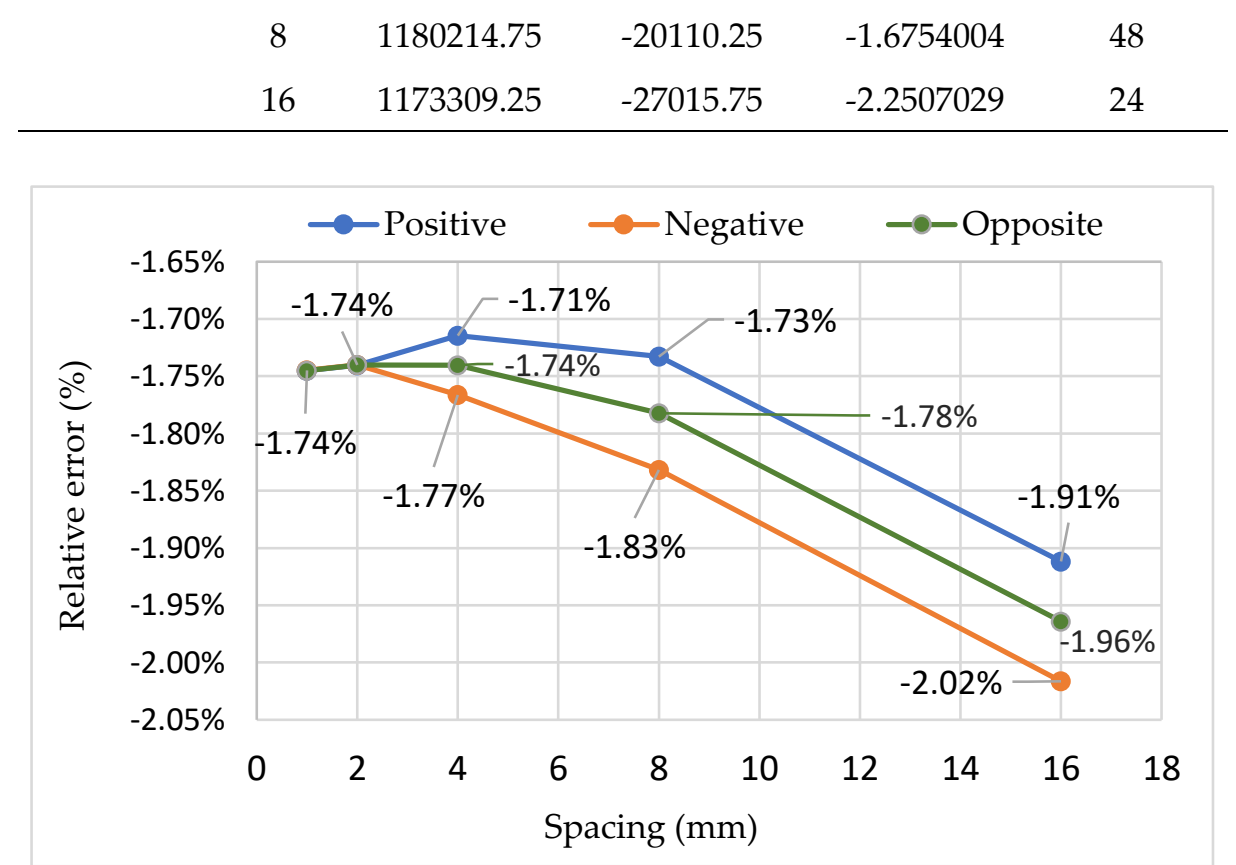

Figure 9. Relative error diagram of volume calculation for oblique axis of stone lion base.

As can be seen from Table 4 and Figure 9, although the volume calculation errors by forward or reverse SM along the oblique axis of the stone lion base are roughly proportional to the slicing spacing, a high volatility and systematicness (low overall) have been observed; these show a marked one-way increasing trend as the spacing increase, which is similar to the Uturn "distortion" of a higher-order polynomial interpolation curve [21]. As a result, the forward and reverse compensatory effects of CODSM are ameliorated to a certain extent and become obvious only when the spacing is small; however, the positive effect of maintaining the calculation results centered and balanced is retained.

From the experiment (as shown in Tables 1-4 and Figures 6-9) and the following analysis, there are great differences in error and compensation between one-way SM and CODSM (as shown in Table 5).

Table 5. Comparison of error and compensation between SM and CODSM.

\begin{tabular}{cccccc}
\hline Object & Method & $\begin{array}{c}\text { Effect of } \\
\text { Random } \\
\text { Errors }\end{array}$ & $\begin{array}{c}\text { Compensation } \\
\text { of } \\
\text { Random } \\
\text { Errors }\end{array}$ & $\begin{array}{c}\text { Effect of } \\
\text { Systematic } \\
\text { Directional } \\
\text { Errors }\end{array}$ & $\begin{array}{c}\text { Compensation } \\
\text { of } \\
\text { Random } \\
\text { Errors }\end{array}$ \\
\hline $\begin{array}{ccccc}\text { Cone } \\
\text { Model }\end{array}$ & SM & Strong & Weak or No & Strong & Weak or No \\
Stone & SM & Weak & Strong & Weak & Strong \\
Lion & Strong & Weak or No & Strong & Weak or No \\
Base & CODSM & $\begin{array}{c}\text { Slightly } \\
\text { Weak }\end{array}$ & Fairly Strong & $\begin{array}{c}\text { Slightly } \\
\text { Weak }\end{array}$ & Fairly Strong \\
\hline
\end{tabular}


From these results, after comprehensively examining and accounting for the fact that "the smaller the slicing spacing, the higher the precision" [14,15], it can be concluded that, overall, CODSM has consistent characteristics and advantages of higher precision, stronger reliability and better robustness than forward or reverse one-way SM.

\section{Discussion}

\subsection{Analysis of Basic Characteristics}

The slicing experiment and calculation of PCO volume of a cone and a stone lion base under two typical working conditions were carried out using SM and CODSM, and the results are shown in Tables 1-4 and Figures 6-9. In general, irrespective of the regularity of PCOs, CODSM used along the positive axis complemented and compensated the best for the errors resulting from shape contingency and directional systematicness, and its advantages are the most distinct. Although a certain degree of weakening and uncertainty in the compensation effect is observed under general working conditions such as oblique axis, the compensation is present in all conditions with the difference being in quantity rather than quality. Consequently, under any given working conditions, the calculation results of CODSM are better, more reliable, and thus more robust than those of the one-way SM.

\subsection{Analysis of the Causes for Compensation}

The root cause for the compensatory effect is that $h_{b}$ (height of the PCO remnant, as shown in Figures 3 and 4) is different for one-way forward and reverse slicing, which results in uncertainties and varying volumes of remnant segments depending on the spacing. Even if the one-way forward and reverse $h_{b}$ happen to be the same, the calculation results obtained by one-way forward and reverse SMs will still vary because of the directionality in the segment volumes. In general, in terms of the segment volume calculation with bottom-up and top-down approaches along the positive axis of the cone, the slices and spacing are tacitly viewed as the bottom and height, respectively, and are used to calculate the volume (see Figure 10); forward or reverse directionality is implied. In other words, when slicing with a bottom-up approach, the volume is calculated upward (as shown in Figure 10a) while with a top-down approach, it is calculated downward (as shown in Figure 10b). Thus, for general irregular PCOs, whether slicing is forward or reverse, the systematic directionality is bound to exert a systematic impact on the volume calculation of the object if the contingency of the slice shape is unknown and uncontrollable. The superposition of random errors and systematic errors coupled with uncertainty will lead to inconsistency in the volume calculation of objects that underwent forward and reverse slicing; furthermore, uncertainty itself is characterized by contingency. Consequently, the CODSM applying the forward and reverse observation and taking the two-way mean as the result can be considered an effective method and a reliable choice to compensate for random and systematic errors. 


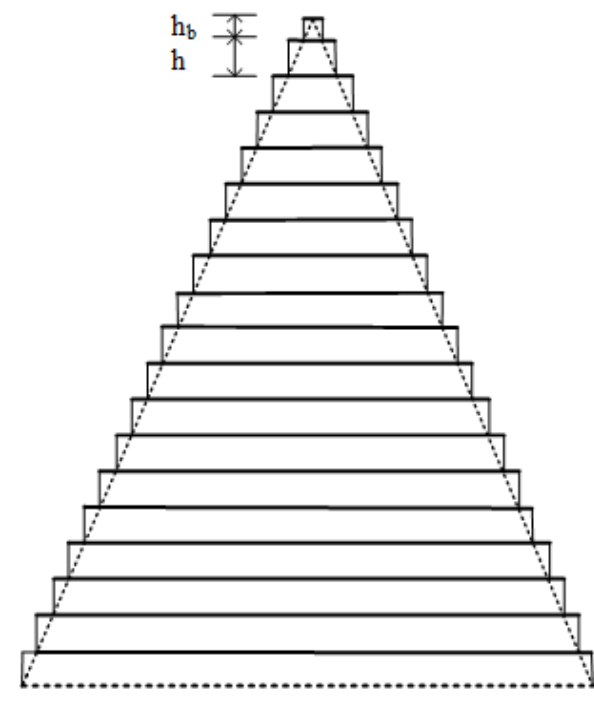

(a)

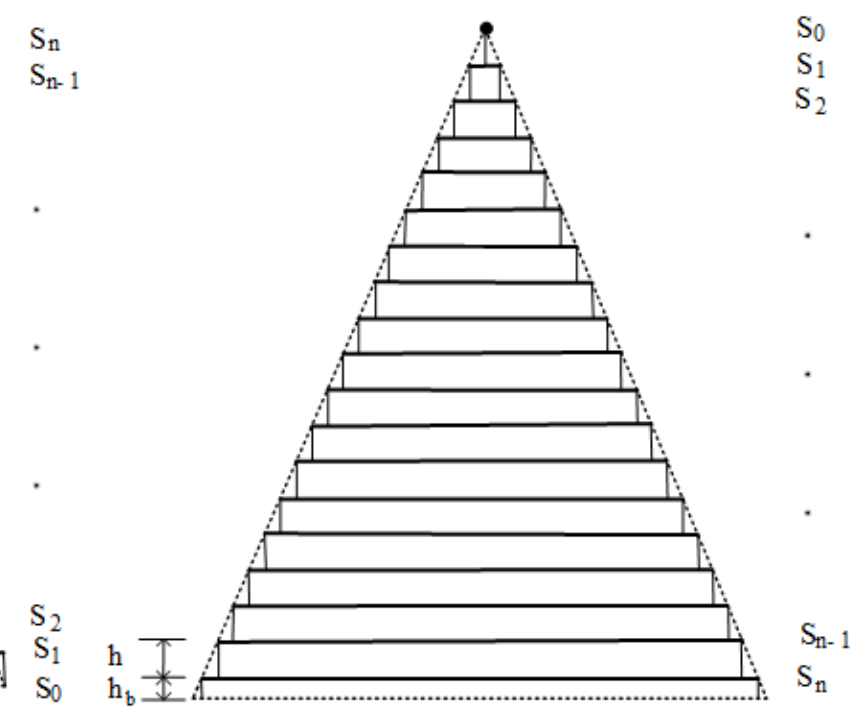

(b)

Figure 10. Schematic of one-way slicing method (taking the positive axis of cone as an example). (a) Slicing from the bottom up; (b) Slicing from the top down.

\subsection{Summary and Prospects}

The CODSM that is proposed and formed based on theoretical analysis and experimentation has been proven effective and has the following characteristics and advantages:

1. The cause and source of the CODSM is a single difference. The differences between forward and reverse SM are in four aspects, namely the slicing position, slices obtained, height of PCO remnant, and PCO remnant itself. The single difference is bound to produce different results for single accumulation, which means, the forward and reverse calculations will lead to systematic deviations.

2. The CODSM can effectively overcome the directionality, volatility, contingency, and systematic nature of SM using co-opposite-direction (i.e., forward and reverse) slicing to deal with directionality. CODSM reduces directional effects, volatility, uncertainty and any systemic effects while partially compensating for possible contingency defects.

3. With better precision compared to the one-way SM when the slicing spacing is small, the CODSM is a more robust and reliable method for PCVC.

4. The CODSM has certain applicable conditions. CODSM has distinct advantages when the spacing is small; however, when the forward and reverse axes are symmetrical (such as the horizontal axis of a cone, the positive axis of a stone lion base, and horizontal axis of a stone lion base), there is little difference between the CODSM and SM; therefore, the latter can be adopted.

5. The CODSM has an advantage range of error compensation; the smaller the spacing, the better the compensatory effect.

6. The error compensation of the CODSM is asymmetric. The asymmetry, which is closely related to the slice shape, varies with working conditions. The ideal working condition is the positive axis of a cone; under other working conditions, different degrees of volatility arise.

7. The errors in the CODSM are positively correlated with the spacing, whether they are random or systematic. Furthermore, the spacing has a certain magnifying effect on these 
errors. For example, during the experiment along the positive axis of the stone lion base with spacings of $4 \mathrm{~mm}, 8 \mathrm{~mm}$, and $16 \mathrm{~mm}$, the relative errors of volume calculation demonstrated volatility (shown in Figure 9), and the relative error openings of forward and reverse slicing display the magnifying feature of increasing with the slicing spacing (shown in Figures 6-9).

8. The CODSM still needs further exploration and expansion. As seen from Figure 7 and Figure 9, it seems to have no solution to the problem that the PCVC along the oblique axis is systematically smaller than the actual value. As shown in Figures 6 and 8, the slice shape is regular and equal in each direction, which is the optimal and preferred working condition along the positive axis in this experiment. As a result, for specific regular or irregular PCOs, an in-depth exploration of the systematic errors of CODSM could be one of the focuses in subsequent research.

\section{Conclusion}

The volatility, systematicness, and contingency of SM are positively correlated with the PCO slicing direction. By increasing parallel observation and considering the two-way mean as the PCO volume, the CODSM proposed in this paper can restrain or even eliminate any uncertain random errors caused by the irregular shape or segment volume of PCO slices, or by the irregular shape of PCOs while essentially weakening the forward, reverse, or unilateral systematic errors of SM. The CODSM is feasible and effective at balancing and reducing both contingency and systematic errors, which makes the calculation results more reasonable and the precision more robust. In conclusion, from the results of the algorithm model and experimental analysis, the CODSM is not only a development from SM, but also a sign indicating that SM can be a full-fledged and reliable method for calculating PCVC.

\section{Funding}

The National Key Research and Development Plan (Grant No. 2018YFC150480505) and Hebei Provincial Geological Environment Monitoring Institute (Grant No. 211526190129) are gratefully acknowledged.

\section{Acknowledgments:}

This study is completed on the basis of SM, an effective method for point cloud object volume calculation. We hereby express our gratitude to actual participants and contributors Li Bin, Wei Junbo, Ma Bochao, Wang Lu in the exploration process of slicing method. We also would like to thank Editage (www.editage.cn) for English language editing.

\section{References}

1. Hillebrand, H.; Dürselen, C.; Kirschtel, D.; Pollingher, U.; Zohary, T. Biovolume calculation for pelagic and benthic microalgae. J PHYCOL 1999, 35, 403-424.

2. Andersen, H.E. Estimation of critical forest structure metrics through the spatial analysis of airborne laser scanner data. International Journal of Simulation: Systems, Science E Techno 2003, 4, 197-202.

3. Benavides-Serralde, A.; Hernández-Andrade, E.; Fernández-Delgado, J.; Plasencia, W.; Scheier, M.; Crispi, F.; Figueras, F.; Nicolaides, K.H.; Gratacós, E. Three-dimensional sonographic calculation of the volume of intracranial 
structures in growth-restricted and appropriate-for-gestational age fetuses. Ultrasound in Obstetrics and Gynecology 2009, 33, 530-537. 10.1002/uog.6343.

4. Wei, X.; Wang, Y.; Zheng, J.; Wang, M.; Feng, Z. Canopy Volume Calculation Method Based on 3 D Laser Scanning Point Cloud. Journal of Agricultural Machinery (in Chinese) 2013, 44, 235-240.

5. Bienert, A.; Hess, C.; Maas, H.G.; Von Oheimb, G. A voxel-based technique to estimate the volume of trees from terrestrial laser scanner data. ISPRS International Archives of the Photogrammetry, Remote Sensing and Spatial Information Sciences 2014, XL-5, 101-106. 10.5194/isprsarchives-XL-5-101-2014.

6. Liu, J.; Xu, Y. A Dissecting and Volume Computing Method For Reservoir Simulation. GEOSCIENCE, Journal of Graduate School, China University of Geosciences 1997, 1997, 217-220.

7. Liu, J.; Wang, W. Forced Convection Tracing Experiment and Volume Calculation of Dual Karst Caves Connected. Journal of Basic Science and Engineering (in Chinese) 2015, 23, 430-438.

8. Douret, J.; Benosman, R. A multi-cameras 3D volumetric method for outdoor scenes: a road traffic monitoring application 2004, 334-337. Cambridge UK

9. Zhang, J.; Cheng, X.; Cheng, X. Chinese Journal of Lasers. Application of 3d Laser Scanning Technology in Measurement of Ship Displacement (in Chinese) 2016, 2016, 162-168.

10. Xie, H.; Gu, F.; Li, Y.; Sun, M. 3 d Modeling Application and Practice Based on Laser Point Cloud Data, Wuhan University Press (in Chinese): Wuhan City, China, 2014; pp 244.

11. Ceng, J.; Zhu, Z.; Ding, R. The way of spatial points reduction and volume calculation based on marching cubes. Surveying science 2008, 33, 81-82.

12. Leveque, R.J. Finite volume methods for hyperbolic problems. MECCANICA 2004, 39, 88-89.

13. Zhi, Y.; Zhang, Y.K.; Chen, H.; Yang, K.F.; Xia, H. A Method of 3D Point Cloud Volume Calculation Based on Slice Method. In ICCA 2016. International Conference on Intelligent Control and Computer Application, Zhengzhou, China, 2016, 155-158.

14. Li, B.; Wei, J.B.; Ma, B.C.; Wang, L.; Xu, M.X. Slicing 3D laser point cloud method for volume calculation of irregular object. Acta Geodaetica et Cartographica Sinica 2019, 48, 42-52.

15. Li, B.; Wei, J.B.; Wang, L.; Ma, B.C.; Xu, M.X. A comparative analysis of two point cloud volume calculation methods. INT J REMOTE SENS 2019, 40, 15-28. 10.1080/01431161.2015.1121301.

16. Tao, B. Statistical theory and method of measurement data processing, Surveying and Mapping Publishing House: Beijing, 2007.

17. Tao, B. Modern adjustment models and its applications. Journal of Nanjing University Information Technology 2009, 1, 27-31. 
18. Liu, D.; Liao, W.; Dai, N.; Cheng, X. Reaearch and implementation for denoising noisy scattered point data. JOURNAL OF SOUTHEAST UNIVERSITY (Natural Science Edition) 2007, 2007, 1108-1112.

19. Pan, R.; Skala, V. Continuous global optimization in surface reconstruction from an oriented point cloud. COMPUT AIDED DESIGN 2011, 43, 896-901.

20. Zhu, J.; Hu, X.; Zhang, Z.; Xiong, X. Hierarchical Outlier Detection for Point Cloud Data Using a Density Analysis Mothod. Acta Geodaetica et Cartographica Sinica 2015, 44, 282-291.

21. Guo, R. Spatial Analysis (2nd edition), Higher education press: Beijing, 2001; pp 255. 